\title{
Estudo descritivo da resposta de acuidade visual sem correção e pressão intra-ocular computadorizada de não contato em jogadores de futebol amadores e profissionais
}

\author{
Emídio Valenti Tavares ${ }^{1}$, Benny Apelbaum ${ }^{1}$, Lysia K. Yukisaki ${ }^{1}$, \\ Yeda Maria Rabboni ${ }^{1}$, Sérgio Barreto Mendes ${ }^{1}$, Odibulho Naves Oliveira ${ }^{1}$, \\ Santos Pedro Tanaganelli ${ }^{1}$ e Francisco Oliveira Rocha ${ }^{2}$ \\ Clínica Oftalmos \& Cirurgia Oftalmológica
}

\section{RESUMO}

O principal objetivo deste estudo foi verificar as possíveis alterações de acuidade visual e pressão intra-ocular em jogadores de futebol. A clínica Oftalmos e Cirurgia Oftalmológica submeteu à avaliação oftalmológica 127 atletas futebolistas provenientes de várias categorias, incluindo mulheres, com idades variando de 14 a 33 anos. Todos foram submetidos a testes de acuidade visual sem correção, utilizando-se a tabela de Snellen e avaliando-se a pressão intra-ocular de não contato com um tonômetro computadorizado (modelo TX-10, $\mathrm{Ca}$ non, EUA). Além disso, os atletas foram submetidos a exame de auto-refração, no equipamento auto-refrator (modelo R30, Canon, EUA). Os seguintes parâmetros utilizados e os resultados encontrados foram: dos 127 atletas avaliados, 56 (44\%) apresentaram alterações de auto-refração, consideradas de grau mais ou menos importante em um ou ambos os olhos. No teste de acuidade visual, 3\% e 2,4\% dos atletas apresentaram, no OD e OE, somente 20/100 e 20/200 pés de visão, respectivamente. Em uma jogadora, foi constatada catarata puntata congênita. Os resultados da tonometria mostraram, em 5 atletas de cor negra, quadro suspeito de glaucoma (necessitando investigação mais apurada). Concluindo, o estudo comprovou a importância da oftalmologia como medida preventiva e/ou corretiva na identificação de possíveis altera-

\footnotetext{
1. Oftalmologista.
}

2. Anestesista.

Endereço para correspondência:

Clínica Oftalmos \& Cirurgia Oftalmológica

Emídio Valenti Tavares

Rua Dr. Coronel Portilho, 75 - Centro

07012-050 - Guarulhos, SP

Tel./fax (0XX11) 6440-1835 ções oftalmológicas em atletas futebolistas, devendo ser uma prática rotineira nesse esporte.

Palavras-chave: Estudo descritivo. Jogador de futebol. Acuidade visual. Pressão intra-ocular. Homens. Mulheres. Medicina esportiva.

\section{ABSTRACT \\ Descriptive study on visual acuity response without correc- tion and computerized non-contact intraocular pressure in amateur and professional (football) soccer players}

The main purpose of this study was to verify possible visual acuity alterations and intraocular pressure in soccer players. An ophthalmological evaluation in 127 soccer athletes belonging to various categories, including female (ages ranging from 14 to 33), was carried out by the Ophthalmologic Clinic Oftalmos. The athletes were all submitted to visual acuity tests without correction, using the Snellen chart and the noncontact intraocular pressure by means of computerized tonometer (TX-model, Canon, USA). They were also submitted to a self-refraction examination using a self refractor $(R-30 \mathrm{mod}$ el, Canon, USA). The parameters used and the results obtained were the following: $56(44 \%)$ of the 127 athletes submitted to evaluation presented self-refractions considered fairly important in one or both eyes. In the visual acuity test $3 \%$ and $2.4 \%$ of the athletes presented in the right and left eye only 20/100 and 20/200 feet of sight, respectively. The presence of a congenital punctate cataract was found in a female player. Tonometry results in five black athletes showed signs of glaucoma, which, however, will require more detailed investigation. In conclusion, the study confirmed the importance of ophthalmology as a preventive and/or corrective measure in order to identify possible ophthalmological alterations in soccer-playing athletes.

Key words: Descriptive study. Soccer players. Visual acuity. Intraocular pressure. Males. Females. Sports medicine. 


\section{INTRODUÇÃO}

A importância de visão dentro dos padrões normais de acuidade é fundamental em atletas, como é o caso de futebolistas, que precisam de reflexo rápido para decidir uma jogada durante o transcorrer de uma partida ${ }^{1}$.

Portanto, avaliar a capacidade de um atleta enxergar o que está à sua frente (visão central) ou dos lados (visão lateral) é de fundamental importância, pois os olhos normais podem reconhecer objetos em uma área de pelo menos $140^{\circ}$ (quase meio circulo).

Se nenhum dos olhos pode ver melhor do que 20/60 pés, sem melhora com o uso de óculos ou lentes corretivas, o atleta pode ser considerado como portador de visão deficitária.

A limitação da visão lateral, visão colorida anormal, visão dupla, visão deficiente à noite e perda de visão em um dos olhos também podem ser consideradas como prejuízo visual.

Uma pessoa com faixa de visão lateral mais estreita pode ter problemas ao andar e reconhecer pessoas em uma sala ampla, mesmo quando a visão central é excelente. Agora, imagine-se um atleta, que precisa decidir uma jogada em fração de segundo. Em jogadores de futebol, a visão lateral é decisiva em muitos lances da partida.

Estudos científicos prospectivos, publicados sobre acuidade visual e pressão intra-ocular em atletas, são escassos, principalmente em jogadores de futebol. Os poucos relatos observados na literatura especializada em medicina esportiva são, em sua grande maioria, sobre prevenção, tratamento, equipamentos e lesões oculares ${ }^{1-15}$.

Portanto, o principal objetivo deste estudo foi mostrar em jogadores de futebol, masculino e feminino, em várias faixas etárias, o perfil oftalmológico de acuidade visual sem correção e pressão intra-ocular desses atletas.

\section{MATERIAL E MÉTODOS}

Foram submetidos à avaliação oftalmológica 127 futebolistas, subdivididos em quatro categorias: infantil (28 jogadores), com média de idade de 14,4 \pm 0,5 anos; juvenil (24 jogadores), com média de idade de 15,9 \pm 0,3 anos; juniores (27 jogadores), com média de idade de 18,4 \pm 1,0 anos; futebolistas femininas (24 jogadoras), com média de idade de 19,7 \pm 4,4 anos e jogadores profissionais (24 atletas), com média de idade de 24,4 \pm 3,3 anos (tabela 1).

A verificação da acuidade visual sem correção, em enxergar objetos na distância longe, foi realizada utilizando-se a escala de Snellen ${ }^{14}$.

O padrão oftalmológico para quantificar a distância de enxergar perto é de $33 \mathrm{~cm}$ e para longe, de $6 \mathrm{~m}$. Na escala de Snellen, o grau de acuidade visual é classificado em pés ou percentagem (\%). Indivíduos com o resultado de 20/20 pés têm $100 \%$ de visão normal para enxergar objetos de longe.

Todos foram ainda submetidos à avaliação da pressão intra-ocular de não contato, que mede a força exercida pelo humor aquoso na câmara anterior (espaço entre a face anterior da íris e a face posterior da córnea) do globo ocular, utilizando-se um tonômetro computadorizado de não contato (modelo TX-10, Canon, EUA).

Os atletas foram submetidos ao exame de auto-refração (refração objetiva), para avaliar possíveis erros refracionais, utilizando-se um auto-refrator computadorizado (modelo R-30, Canon, EUA).

\section{RESULTADOS}

Os resultados observados neste estudo são apresentados por categoria e dados individuais de cada atleta, listados nas tabelas $1,2,3,4,5,6$ e 7 .

\section{DISCUSSÃO E COMENTÁRIOS}

A participação preventiva e investigativa da oftalmologia na medicina do esporte e, especificamente, no futebol, em nosso país, tem sido escassa. É difícil determinar razão para tal fato. Entretanto, parece que a falta de esclarecimento sobre a importância dos benefícios dessa área para o esporte e, sobretudo, o atleta, seja a causa principal.

A grande maioria dos estudos tem-se preocupado em relatar lesões oculares, relacionadas com vários tipos de esportes,

\begin{tabular}{|c|c|c|c|c|c|c|}
\hline \multicolumn{7}{|c|}{$\begin{array}{l}\text { TABELA } 1 \\
\text { Distribuição de todos os futebolistas por idade, } n \text { o de jogadores } \\
\text { e categoria, submetidos à avaliação oftalmológica }(n=127)\end{array}$} \\
\hline & Total & Infantil & J uvenil & J uniores & Profissionais & Mulheres \\
\hline & $\begin{array}{l}\text { Homens/ } \\
\text { mulheres }\end{array}$ & Homens & Homens & Homens & Homens & Futebolistas \\
\hline № & 127 & 28 & 24 & 27 & 24 & 24 \\
\hline Idade & 18,5 & 14,4 & 15,9 & 18,4 & 24,4 & 19,7 \\
\hline (anos) & $\pm 3,8$ & $\pm 0,5$ & $\pm 0,3$ & $\pm 1,0$ & $\pm 3,3$ & $\pm 4,4$ \\
\hline
\end{tabular}


atividades recreativas, equipamentos, prevenção e com os aspectos epidemiológicos do problema ${ }^{1-15}$.

Recentemente, a Academia Americana de Oftalmologia ${ }^{16}$ classificou algumas modalidades esportivas pela popularidade e a alta incidência de trauma ocular. Foi constatado que o beisebol e o basquetebol foram as modalidades associadas ao maior número de lesões em atletas entre 5 e 24 anos de idade 9 .

O futebol (soccer) foi classificado em sétimo lugar. Entretanto, essa entidade não informou sobre a gravidade das lesões oculares.

Em outro estudo publicado por MacEwen ${ }^{5}$ foram verificados em 246 indivíduos diversos tipos de lesões. Destas, 110 $(44,7 \%)$ foram em jogadores de futebol, 24 (9,8\%) no rúgbi, $16(6,5 \%)$ no badminton e $68(27,6 \%)$ em outros esportes.

Barrell et al. ${ }^{6}$, ao atenderem 118 indivíduos, constataram que a freqüência de lesões foi proveniente do squash, badminton, tênis de campo, tênis de mesa, críquete e futebol. Nesse estudo, a incidência maior de lesões foi provocada pelo squash.

Ao contrário, Filipe et al. ${ }^{7}$, ao avaliarem 84 pacientes com lesões esportivas por um período de três anos, verificaram que as modalidades esportivas responsáveis pela maior freqüên-

\begin{tabular}{|c|c|c|c|c|c|c|}
\hline \multicolumn{7}{|c|}{$\begin{array}{c}\text { TABELA } 2 \\
\text { Medidas individuais de distribuição de acuidade visual } \\
\text { sem correção e pressão intra-ocular dos olhos } \\
\text { direito (OD) e esquerdo (OE) em jogadores } \\
\text { de futebol - categoria profissional }(n=24)\end{array}$} \\
\hline \multirow[t]{2}{*}{ № } & \multirow{2}{*}{$\begin{array}{l}\text { Sexo } \\
(\mathrm{m} / \mathrm{f})\end{array}$} & \multirow{2}{*}{$\begin{array}{l}\text { Idade } \\
\text { (anos) }\end{array}$} & \multicolumn{2}{|c|}{ Acuidade visual } & \multicolumn{2}{|c|}{ Pressão intra-ocular } \\
\hline & & & $\begin{array}{l}\text { OD } \\
\text { (pés) }\end{array}$ & $\begin{array}{c}\text { OE } \\
\text { (pés) }\end{array}$ & $\begin{array}{c}\text { OD } \\
(\mathrm{mmHg})\end{array}$ & $\begin{array}{c}\mathrm{OE} \\
(\mathrm{mmHg})\end{array}$ \\
\hline 1 & $\mathrm{~m}$ & 29 & $20 / 20$ & $20 / 25$ & 9,6 & 11,5 \\
\hline 2 & $\mathrm{~m}$ & 29 & $20 / 20$ & $20 / 200$ & 13,2 & 12,6 \\
\hline 3 & $\mathrm{~m}$ & 27 & $20 / 20$ & $20 / 20$ & 10,3 & 13,0 \\
\hline 4 & $\mathrm{~m}$ & 24 & $20 / 20$ & $20 / 20$ & 7,7 & 8,1 \\
\hline 5 & $\mathrm{~m}$ & 20 & $20 / 20$ & $20 / 20$ & 17,7 & 19,3 \\
\hline 6 & $\mathrm{~m}$ & 23 & $20 / 20$ & $20 / 20$ & 14,4 & 18,4 \\
\hline 7 & $\mathrm{~m}$ & 29 & $20 / 20$ & $20 / 20$ & 20,6 & 16,9 \\
\hline 8 & $\mathrm{~m}$ & 28 & $20 / 20$ & $20 / 20$ & 11,2 & 10,5 \\
\hline 9 & $\mathrm{~m}$ & 25 & $20 / 20$ & $20 / 20$ & 9,3 & 9,2 \\
\hline 10 & $\mathrm{~m}$ & 24 & $20 / 20$ & $20 / 20$ & 15,9 & 14,3 \\
\hline 11 & $\mathrm{~m}$ & 22 & $20 / 20$ & $20 / 20$ & 9,9 & 8,8 \\
\hline 12 & $\mathrm{~m}$ & 21 & $20 / 20$ & $20 / 20$ & 10,7 & 12,6 \\
\hline 13 & $\mathrm{~m}$ & 21 & $20 / 20$ & $20 / 20$ & 7,0 & 6,7 \\
\hline 14 & $\mathrm{~m}$ & 22 & $20 / 20$ & $20 / 20$ & 12,8 & 9,4 \\
\hline 15 & $\mathrm{~m}$ & 21 & $20 / 20$ & $20 / 20$ & 10,7 & 10,7 \\
\hline 16 & $\mathrm{~m}$ & 25 & $20 / 30$ & $20 / 30$ & 13,0 & 13,8 \\
\hline 17 & $\mathrm{~m}$ & 23 & $20 / 20$ & $20 / 20$ & 7,7 & 7,1 \\
\hline 18 & $\mathrm{~m}$ & 33 & $20 / 20$ & $20 / 20$ & 7,3 & 9,0 \\
\hline 19 & $\mathrm{~m}$ & 23 & $20 / 20$ & $20 / 20$ & 11,4 & 11,9 \\
\hline 20 & $\mathrm{~m}$ & 22 & $20 / 20$ & $20 / 20$ & 14,0 & 16,0 \\
\hline 21 & $m$ & 27 & $20 / 20$ & $20 / 20$ & 12,2 & 14,0 \\
\hline 22 & $\mathrm{~m}$ & 23 & $20 / 20$ & $20 / 20$ & 19,0 & 12,0 \\
\hline 23 & $\mathrm{~m}$ & 24 & $20 / 25$ & $20 / 30$ & 12,5 & 12,4 \\
\hline 24 & $\mathrm{~m}$ & 21 & $20 / 20$ & $20 / 20$ & 12,6 & 10,4 \\
\hline
\end{tabular}

cia de traumas oculares foram, respectivamente, o futebol outdoor (campo) e o indoor (salão) (72,6\% dos casos).

O tipo mais freqüiente de lesão foi o hifema (depósito de sangue entre a parte posterior da córnea e a parte anterior da íris). Dos 45 indivíduos que apresentaram esse quadro, 24 $(53,3 \%)$ tinham lesões vítreas e/ou na retina. Quando se comparou com indivíduos sem hifema, a percentagem caiu para $33,3 \%$. Em 19 indivíduos dos 58 avaliados $(32,8 \%)$ com acuidade visual normal ( $\geq 20 / 40$ pés), a causa mais comum foi a lesão vítrea ou retiniana. No futebol, o trauma mais freqüente encontrado por eles foi o hifema.

Apesar da importante contribuição desses estudos, não encontramos nenhum relato na literatura mostrando o perfil de acuidade visual e/ou pressão intra-ocular em jogadores de futebol.

No presente estudo, pudemos verificar alterações oculares importantes em atletas futebolistas (tabela 7). Em alguns casos, a pressão intra-ocular se mostrou elevada, sendo necessária a investigação de possível quadro glaucomatoso familiar.

TABELA 3

Medidas individuais de distribuição de acuidade visual sem correção e pressão intra-ocular dos olhos direito (OD) e esquerdo (OE) em jogadores de futebol - categoria infantil $(n=28)$

\begin{tabular}{|c|c|c|c|c|c|c|}
\hline \multirow[t]{2}{*}{ № } & \multirow{2}{*}{$\begin{array}{l}\text { Sexo } \\
(\mathrm{m} / \mathrm{f})\end{array}$} & \multirow{2}{*}{$\begin{array}{l}\text { Idade } \\
\text { (anos) }\end{array}$} & \multicolumn{2}{|c|}{ Acuidade visual } & \multicolumn{2}{|c|}{ Pressão intra-ocular } \\
\hline & & & $\begin{array}{l}\text { OD } \\
\text { (pés) }\end{array}$ & $\begin{array}{l}\text { OE } \\
\text { (pés) }\end{array}$ & $\begin{array}{c}\text { OD } \\
(\mathrm{mmHg})\end{array}$ & $\begin{array}{c}\mathrm{OE} \\
(\mathrm{mmHg})\end{array}$ \\
\hline 1 & $\mathrm{~m}$ & 14 & $20 / 20$ & $20 / 20$ & 11,0 & 10,8 \\
\hline 2 & $\mathrm{~m}$ & 14 & $20 / 20$ & $20 / 20$ & 13,4 & 13,7 \\
\hline 3 & $\mathrm{~m}$ & 14 & $20 / 25$ & $20 / 20$ & 10,4 & 12,1 \\
\hline 4 & $\mathrm{~m}$ & 14 & $20 / 20$ & $20 / 20$ & 10,6 & 12,4 \\
\hline 5 & $\mathrm{~m}$ & 15 & $20 / 20$ & $20 / 20$ & 8,7 & 9,1 \\
\hline 6 & $\mathrm{~m}$ & 15 & $20 / 20$ & $20 / 20$ & 9,6 & 12,1 \\
\hline 7 & $\mathrm{~m}$ & 15 & $20 / 20$ & $20 / 20$ & 17,7 & 17,7 \\
\hline 8 & $\mathrm{~m}$ & 15 & $20 / 20$ & $20 / 20$ & 12,1 & 12,2 \\
\hline 9 & $\mathrm{~m}$ & 14 & $20 / 200$ & $20 / 20$ & 13,0 & 11,5 \\
\hline 10 & $\mathrm{~m}$ & 15 & $20 / 40$ & $20 / 20$ & 14,1 & 12,4 \\
\hline 11 & $\mathrm{~m}$ & 14 & $20 / 20$ & $20 / 20$ & 10,7 & 13,1 \\
\hline 12 & $\mathrm{~m}$ & 15 & $20 / 20$ & $20 / 20$ & 12,8 & 12,5 \\
\hline 13 & $\mathrm{~m}$ & 14 & $20 / 20$ & $20 / 20$ & 13,2 & 13,6 \\
\hline 14 & $\mathrm{~m}$ & 14 & $20 / 20$ & $20 / 25$ & 14,3 & 14,3 \\
\hline 15 & $\mathrm{~m}$ & 14 & $20 / 20$ & $20 / 20$ & 13,4 & 14,1 \\
\hline 16 & $\mathrm{~m}$ & 15 & $20 / 20$ & $20 / 20$ & 13,3 & 11,9 \\
\hline 17 & $\mathrm{~m}$ & 14 & $20 / 20$ & $20 / 20$ & 16,9 & 17,1 \\
\hline 18 & $\mathrm{~m}$ & 14 & $20 / 20$ & $20 / 20$ & 15,9 & 16,6 \\
\hline 19 & $\mathrm{~m}$ & 14 & $20 / 20$ & $20 / 20$ & 14,3 & 15,9 \\
\hline 20 & $\mathrm{~m}$ & 15 & $20 / 20$ & $20 / 20$ & 15,4 & 13,6 \\
\hline 21 & $\mathrm{~m}$ & 15 & $20 / 20$ & $20 / 20$ & 11,2 & 13,2 \\
\hline 22 & $\mathrm{~m}$ & 14 & $20 / 20$ & $20 / 20$ & 11,2 & 14,3 \\
\hline 23 & $\mathrm{~m}$ & 15 & $20 / 20$ & $20 / 20$ & 16,7 & 11,6 \\
\hline 24 & $\mathrm{~m}$ & 14 & $20 / 20$ & $20 / 20$ & 16,2 & 11,9 \\
\hline 25 & $\mathrm{~m}$ & 14 & $20 / 20$ & $20 / 20$ & 10,0 & 10,9 \\
\hline 26 & $\mathrm{~m}$ & 15 & $20 / 200$ & $20 / 200$ & 15,8 & 13,8 \\
\hline 27 & $\mathrm{~m}$ & 14 & $20 / 20$ & $20 / 20$ & 16,8 & 15,1 \\
\hline 28 & $\mathrm{~m}$ & 15 & $20 / 20$ & $20 / 20$ & 12,8 & 13,0 \\
\hline
\end{tabular}


Em outro caso, um futebolista da categoria infantil, com 14 anos de idade, apresentou no olho direito (OD) acuidade visual de 20/200 pés. Quando submetido ao exame de auto-refração computadorizada, apresentou ambliopia ex-anopsia refracional (olho preguiçoso) em OD de $+1,00^{\circ} \mathrm{DE}$ (hipermetropia) com $-5,50^{\circ} \mathrm{DC}$ (astigmatismo). Já o olho esquerdo (OE) apresentou-se plano (emetrope). A pressão intra-ocular em ambos os olhos foi de 13,0 e 15,0 $\mathrm{mmHg}$, respectivamente, considerada normal.

Em mais um caso verificado na categoria infantil, um atleta apresentou visão de 20/200 AO. Entretanto, a pressão intraocular foi considerada normal, ou seja, 15,8 e 13,8mmHg em ambos os olhos, respectivamente. Contudo, o teste de autorefração mostrou em ambos os olhos $-1,50^{\circ} \mathrm{DE}$ com $-0,25^{\circ}$ DC.

Dois jogadores juniores apresentaram astigmatismo em OD de $-1,25^{\circ}$ e $-2,50^{\circ}$ dioptrias cilíndricas (DC), respectivamente. Nesses atletas não foi verificada ambliopia e a tonometria era normal em ambos os olhos.

\begin{tabular}{|c|c|c|c|c|c|c|}
\hline \multirow{3}{*}{ № } & \multicolumn{6}{|c|}{$\begin{array}{c}\text { TABELA } 4 \\
\text { Medidas individuais de distribuição de acuidade visual } \\
\text { sem correção e pressão intra-ocular dos olhos } \\
\text { direito (OD) e esquerdo (OE) em jogadores } \\
\text { de futebol - categoria juniores }(\mathbf{n}=\mathbf{2 7})\end{array}$} \\
\hline & \multirow{2}{*}{$\begin{array}{l}\text { Sexo } \\
(m / f)\end{array}$} & \multirow{2}{*}{$\begin{array}{l}\text { Idade } \\
\text { (anos) }\end{array}$} & \multicolumn{2}{|c|}{ Acuidade visual } & \multicolumn{2}{|c|}{ Pressão intra-ocular } \\
\hline & & & $\begin{array}{l}\text { OD } \\
\text { (pés) }\end{array}$ & $\begin{array}{l}\text { OE } \\
\text { (pés) }\end{array}$ & $\begin{array}{c}\text { OD } \\
(\mathrm{mmHg})\end{array}$ & $\begin{array}{c}\mathrm{OE} \\
(\mathrm{mmHg})\end{array}$ \\
\hline 1 & $\mathrm{~m}$ & 19 & $20 / 20$ & $20 / 20$ & 12,5 & 8,5 \\
\hline 2 & $\mathrm{~m}$ & 17 & $20 / 20$ & $20 / 20$ & 16,2 & 14,1 \\
\hline 3 & $\mathrm{~m}$ & 18 & $20 / 20$ & $20 / 20$ & 8,7 & 9,4 \\
\hline 4 & $\mathrm{~m}$ & 20 & $20 / 20$ & $20 / 20$ & 13,3 & 10,7 \\
\hline 5 & $\mathrm{~m}$ & 18 & $20 / 20$ & $20 / 20$ & 15,1 & 15,9 \\
\hline 6 & $\mathrm{~m}$ & 18 & $20 / 20$ & $20 / 25$ & 11,2 & 11,2 \\
\hline 7 & $\mathrm{~m}$ & 19 & $20 / 20$ & $20 / 20$ & 12,5 & 15,4 \\
\hline 8 & $\mathrm{~m}$ & 18 & $20 / 20$ & $20 / 20$ & 17,3 & 13,8 \\
\hline 9 & $\mathrm{~m}$ & 17 & $20 / 20$ & $20 / 25$ & 19,7 & 19,1 \\
\hline 10 & $\mathrm{~m}$ & 19 & $20 / 20$ & $20 / 20$ & 11,2 & 11,8 \\
\hline 11 & $\mathrm{~m}$ & 18 & $20 / 20$ & $20 / 20$ & 13,4 & 12,8 \\
\hline 12 & $\mathrm{~m}$ & 19 & $20 / 20$ & $20 / 20$ & 14,8 & 13,7 \\
\hline 13 & $\mathrm{~m}$ & 19 & $20 / 20$ & $20 / 20$ & 19,3 & 19,9 \\
\hline 14 & $\mathrm{~m}$ & 17 & $20 / 20$ & $20 / 20$ & 11,6 & 14,0 \\
\hline 15 & $\mathrm{~m}$ & 20 & $20 / 20$ & $20 / 20$ & 12,7 & 12,9 \\
\hline 16 & $\mathrm{~m}$ & 19 & $20 / 20$ & $20 / 20$ & 15,0 & 10,8 \\
\hline 17 & $\mathrm{~m}$ & 17 & $20 / 20$ & $20 / 20$ & 17,8 & 20,4 \\
\hline 18 & $\mathrm{~m}$ & 17 & $20 / 20$ & $20 / 20$ & 12,7 & 17,8 \\
\hline 19 & $\mathrm{~m}$ & 18 & $20 / 20$ & $20 / 20$ & 12,0 & 12,0 \\
\hline 20 & $m$ & 18 & $20 / 60$ & $20 / 40$ & 14,4 & 11,2 \\
\hline 21 & $\mathrm{~m}$ & 20 & $20 / 20$ & $20 / 20$ & 12,8 & 14,2 \\
\hline 22 & $\mathrm{~m}$ & 19 & $20 / 20$ & $20 / 20$ & 8,4 & 9,4 \\
\hline 23 & $\mathrm{~m}$ & 20 & $20 / 20$ & $20 / 20$ & 18,7 & 18,7 \\
\hline 24 & $\mathrm{~m}$ & 19 & $20 / 40$ & $20 / 30$ & 12,9 & 10,6 \\
\hline 25 & $\mathrm{~m}$ & 19 & $20 / 20$ & $20 / 20$ & 9,2 & 7,7 \\
\hline 26 & $\mathrm{~m}$ & 18 & $20 / 20$ & $20 / 20$ & 13,2 & 15,4 \\
\hline 27 & $\mathrm{~m}$ & 17 & $20 / 20$ & $20 / 20$ & 9,0 & 10,1 \\
\hline
\end{tabular}

TABELA 5

Medidas individuais de distribuição de acuidade visual sem correção e pressão intra-ocular dos olhos direito (OD) e esquerdo (OE) em jogadoras de futebol $(n=24)$

\begin{tabular}{|c|c|c|c|c|c|c|}
\hline \multirow[t]{2}{*}{ № } & \multirow{2}{*}{$\begin{array}{l}\text { Sexo } \\
(\mathrm{m} / \mathrm{f})\end{array}$} & \multirow{2}{*}{$\begin{array}{l}\text { Idade } \\
\text { (anos) }\end{array}$} & \multicolumn{2}{|c|}{ Acuidade visual } & \multicolumn{2}{|c|}{ Pressão intra-ocular } \\
\hline & & & $\begin{array}{l}\text { OD } \\
\text { (pés) }\end{array}$ & $\begin{array}{l}\text { OE } \\
\text { (pés) }\end{array}$ & $\begin{array}{c}\text { OD } \\
(\mathrm{mmHg})\end{array}$ & $\begin{array}{c}\mathrm{OE} \\
(\mathrm{mmHg})\end{array}$ \\
\hline 1 & $f$ & 19 & $20 / 20$ & $20 / 20$ & 13,0 & 12,0 \\
\hline 2 & $f$ & 19 & $20 / 20$ & $20 / 20$ & 16,0 & 17,0 \\
\hline 3 & $f$ & 30 & $20 / 20$ & $20 / 20$ & 17,0 & 14,0 \\
\hline 4 & $f$ & 23 & $20 / 20$ & $20 / 20$ & 13,2 & 12,5 \\
\hline 5 & $f$ & 14 & $20 / 20$ & $20 / 20$ & 11,6 & 14,4 \\
\hline 6 & f & 19 & $20 / 20$ & $20 / 20$ & 9,9 & 14,0 \\
\hline 7 & $f$ & 16 & $20 / 20$ & $20 / 20$ & 12,2 & 10,8 \\
\hline 8 & $f$ & 19 & $20 / 20$ & $20 / 20$ & 12,3 & 10,8 \\
\hline 9 & $\mathrm{f}$ & 16 & $20 / 20$ & $20 / 20$ & 10,1 & 12,8 \\
\hline 10 & $f$ & 23 & $20 / 25$ & $20 / 25$ & 14,8 & 10,5 \\
\hline 11 & $f$ & 23 & $20 / 25$ & $20 / 25$ & 16,1 & 11,1 \\
\hline 12 & $\mathrm{f}$ & 16 & $20 / 30$ & $20 / 30$ & 18,4 & 17,8 \\
\hline 13 & $f$ & 34 & $20 / 20$ & $20 / 20$ & 12,5 & 12,6 \\
\hline 14 & $\mathrm{f}$ & 32 & $20 / 20$ & $20 / 20$ & 15,9 & 17,8 \\
\hline 15 & $f$ & 21 & $20 / 100$ & 20/100 & 19,5 & 19,2 \\
\hline 16 & $f$ & 30 & $20 / 20$ & $20 / 20$ & 9,3 & 9,2 \\
\hline 17 & $f$ & 20 & $20 / 25$ & $20 / 25$ & 23,0 & 19,8 \\
\hline 18 & $f$ & 17 & $20 / 20$ & $20 / 20$ & 17,5 & 20,5 \\
\hline 19 & $f$ & 22 & $20 / 30$ & $20 / 30$ & 19,7 & 16,9 \\
\hline 20 & f & 19 & $20 / 30$ & $20 / 20$ & 19,1 & 13,4 \\
\hline 21 & f & 21 & $20 / 20$ & $20 / 20$ & 20,8 & 20,5 \\
\hline 22 & f & 16 & $20 / 20$ & $20 / 20$ & 13,5 & 14,1 \\
\hline 23 & $f$ & 17 & $20 / 20$ & $20 / 20$ & 16,3 & 19,0 \\
\hline 24 & $f$ & 16 & $20 / 30$ & $20 / 30$ & 15,2 & 10,6 \\
\hline
\end{tabular}

TABELA 6

Medidas individuais de acuidade visual sem correção e pressão intra-ocular dos olhos direito (OD) e esquerdo (OE) em jogadores de futebol - categoria juvenil $(n=24)$

\begin{tabular}{|c|c|c|c|c|c|c|}
\hline \multirow[t]{2}{*}{ № } & \multirow{2}{*}{$\begin{array}{l}\text { Sexo } \\
(\mathrm{m} / \mathrm{f})\end{array}$} & \multirow{2}{*}{$\begin{array}{l}\text { Idade } \\
\text { (anos) }\end{array}$} & \multicolumn{2}{|c|}{ Acuidade visual } & \multicolumn{2}{|c|}{ Pressão intra-ocular } \\
\hline & & & $\begin{array}{l}\text { OD } \\
\text { (pés) }\end{array}$ & $\begin{array}{l}\text { OE } \\
\text { (pés) }\end{array}$ & $\begin{array}{c}\text { OD } \\
(\mathbf{m m H g})\end{array}$ & $\begin{array}{c}\mathrm{OE} \\
(\mathrm{mmHg})\end{array}$ \\
\hline 1 & $\mathrm{~m}$ & 16 & $20 / 20$ & $20 / 20$ & 15,5 & 15,3 \\
\hline 2 & $\mathrm{~m}$ & 16 & $20 / 20$ & $20 / 20$ & 120 & 10,5 \\
\hline 3 & $\mathrm{~m}$ & 15 & $20 / 20$ & $20 / 25$ & 15,5 & 11,9 \\
\hline 4 & $\mathrm{~m}$ & 16 & $20 / 20$ & $20 / 20$ & 12,7 & 13,5 \\
\hline 5 & $\mathrm{~m}$ & 16 & $20 / 20$ & $20 / 20$ & 12,9 & 11,7 \\
\hline 6 & $\mathrm{~m}$ & 16 & $20 / 20$ & $20 / 20$ & 11,1 & 11,2 \\
\hline 7 & $\mathrm{~m}$ & 16 & $20 / 20$ & $20 / 20$ & 14,1 & 12,8 \\
\hline 8 & $\mathrm{~m}$ & 16 & $20 / 20$ & $20 / 20$ & 14,3 & 15,2 \\
\hline 9 & $\mathrm{~m}$ & 16 & $20 / 200$ & $20 / 200$ & 19,0 & 16.2 \\
\hline 10 & $\mathrm{~m}$ & 16 & $20 / 20$ & $20 / 20$ & 16,7 & 14,2 \\
\hline 11 & $\mathrm{~m}$ & 16 & $20 / 20$ & $20 / 25$ & 11,0 & 11,9 \\
\hline 12 & $\mathrm{~m}$ & 16 & $20 / 25$ & $20 / 25$ & 11,9 & 11,5 \\
\hline 13 & $\mathrm{~m}$ & 16 & $20 / 20$ & $20 / 20$ & 8,9 & 8,9 \\
\hline 14 & $\mathrm{~m}$ & 17 & $20 / 20$ & $20 / 20$ & 6,4 & 8,2 \\
\hline 15 & $\mathrm{~m}$ & 15 & $20 / 20$ & $20 / 20$ & 15,7 & 14,4 \\
\hline 16 & $\mathrm{~m}$ & 16 & $20 / 20$ & $20 / 20$ & 12,3 & 13,2 \\
\hline 17 & $\mathrm{~m}$ & 16 & $20 / 20$ & $20 / 20$ & 16,2 & 15,8 \\
\hline 18 & $\mathrm{~m}$ & 16 & $20 / 20$ & $20 / 20$ & 12,1 & 10,9 \\
\hline 19 & $\mathrm{~m}$ & 16 & $20 / 20$ & $20 / 20$ & 11,8 & 15,0 \\
\hline 20 & $\mathrm{~m}$ & 16 & $20 / 20$ & $20 / 20$ & 15,2 & 14,1 \\
\hline 21 & $\mathrm{~m}$ & 16 & $20 / 20$ & $20 / 20$ & 11,2 & 11,5 \\
\hline 22 & $\mathrm{~m}$ & 16 & $20 / 20$ & $20 / 30$ & 8,9 & 12,5 \\
\hline 23 & $\mathrm{~m}$ & 16 & $20 / 20$ & $20 / 20$ & 12,2 & 9,0 \\
\hline 24 & $\mathrm{~m}$ & 16 & $20 / 20$ & $20 / 25$ & 16,3 & 14,7 \\
\hline
\end{tabular}




\begin{tabular}{|c|c|c|c|c|c|c|c|}
\hline \multirow{3}{*}{ № } & \multicolumn{7}{|c|}{$\begin{array}{l}\text { TABELA } 7 \\
\text { Medidas individuais de distribuição de auto-refração computadorizada dos olhos } \\
\text { direito (OD) e esquerdo (OE) em jogadores de futebol feminino e masculino }(n=56)\end{array}$} \\
\hline & \multirow[t]{2}{*}{$\operatorname{Sexo}(m / f)$} & \multirow[t]{2}{*}{ Idade (anos) } & \multirow[t]{2}{*}{ Posição } & \multicolumn{2}{|c|}{ OD } & \multicolumn{2}{|c|}{ OE } \\
\hline & & & & Esféricas & Cilíndricas & Esféricas & Cilíndricas \\
\hline 1 & $f$ & 16 & Goleira & $+0,75 \mathrm{D}$ & $-2,50 \mathrm{D}$ & $+0,50 \mathrm{D}$ & $-1,75 \mathrm{D}$ \\
\hline 2 & $\mathrm{~m}$ & 14 & & $+1,00 \mathrm{D}$ & $-5,50 \mathrm{D}$ & 0,00 & 0,00 \\
\hline 3 & $\mathrm{~m}$ & 19 & Meio-campo & 0,00 & $-1,25 \mathrm{D}$ & $-0,50 \mathrm{D}$ & $-0,75 \mathrm{D}$ \\
\hline 4 & $\mathrm{~m}$ & 18 & Lateral & 0,00 & $-2,50 \mathrm{D}$ & $-0,25 \mathrm{D}$ & $-1,75 \mathrm{D}$ \\
\hline 5 & $\mathrm{~m}$ & 19 & & $+0,50 \mathrm{D}$ & $-0,50 \mathrm{D}$ & 0,00 & $-0,75 \mathrm{D}$ \\
\hline 6 & $\mathrm{~m}$ & 18 & Meio-campo & 0,00 & $-0,50 \mathrm{D}$ & 0,00 & $-0,75 \mathrm{D}$ \\
\hline 7 & $\mathrm{~m}$ & 17 & & $-0,25 \mathrm{D}$ & $-0,75 \mathrm{D}$ & $-0,25 \mathrm{D}$ & $-0,75 \mathrm{D}$ \\
\hline 8 & $f$ & 21 & Atacante & $+0,25 \mathrm{D}$ & $-1,50 \mathrm{D}$ & $+0,50 \mathrm{D}$ & $-1,25 \mathrm{D}$ \\
\hline 9 & $f$ & 20 & Meio-campo & $+0,50 \mathrm{D}$ & $-1,00 \mathrm{D}$ & $+0,50 \mathrm{D}$ & $-1,00 \mathrm{D}$ \\
\hline 10 & $f$ & 22 & M eio-campo & $-0,50 \mathrm{D}$ & $-0,50 \mathrm{D}$ & $-0,50 \mathrm{D}$ & $-0,50 \mathrm{D}$ \\
\hline 11 & $f$ & 16 & Meio-campo & $-0,50 \mathrm{D}$ & $-1,00 \mathrm{D}$ & $-0,25 \mathrm{D}$ & $-1,00 \mathrm{D}$ \\
\hline 12 & $\mathrm{~m}$ & 16 & Atacante & $+0,25 \mathrm{D}$ & $-0,50 \mathrm{D}$ & Plano & $-0,50 \mathrm{D}$ \\
\hline 13 & $\mathrm{~m}$ & 16 & Lateral & $-0,25 \mathrm{D}$ & $-0,25 \mathrm{D}$ & $-0,50 \mathrm{D}$ & $-0,50 \mathrm{D}$ \\
\hline 14 & $\mathrm{~m}$ & 15 & Atacante & & & Plano & $-0,25 \mathrm{D}$ \\
\hline 15 & $\mathrm{~m}$ & 16 & Meio-campo & & & Plano & $-0,25 \mathrm{D}$ \\
\hline 16 & $\mathrm{~m}$ & 16 & Atacante & Plano & $-0,25 \mathrm{D}$ & Plano & $-0,25 \mathrm{D}$ \\
\hline 17 & $\mathrm{~m}$ & 16 & Defesa & $+0,25 \mathrm{D}$ & $+0,25 \mathrm{D}$ & Plano & $-0,25 \mathrm{D}$ \\
\hline 18 & $\mathrm{~m}$ & 16 & Meio-campo & $+0,25 \mathrm{D}$ & $-0,50 \mathrm{D}$ & & \\
\hline 19 & $\mathrm{~m}$ & 16 & Lateral & $-2,25 \mathrm{D}$ & $-0,50 \mathrm{D}$ & $-1,50 \mathrm{D}$ & $-1,50 \mathrm{D}$ \\
\hline 20 & $\mathrm{~m}$ & 16 & M eio-campo & $+0,75 \mathrm{D}$ & $-0,25 \mathrm{D}$ & $+0,75 \mathrm{D}$ & \\
\hline 21 & $\mathrm{~m}$ & 16 & M eio-campo & & & Plano & $-1,25 \mathrm{D}$ \\
\hline 22 & $\mathrm{~m}$ & 16 & Meio-campo & $-0,50 \mathrm{D}$ & & $-0,25 \mathrm{D}$ & $-0,50 \mathrm{D}$ \\
\hline 23 & $\mathrm{~m}$ & 16 & Atacante & $+0,75 \mathrm{D}$ & & $+0,75 \mathrm{D}$ & \\
\hline 24 & $\mathrm{~m}$ & 17 & Defesa & Plano & $-0,75 \mathrm{D}$ & Plano & $-0,50 \mathrm{D}$ \\
\hline 25 & $\mathrm{~m}$ & 15 & Defesa & Plano & & $+0,25 \mathrm{D}$ & $-0,25 \mathrm{D}$ \\
\hline 26 & $\mathrm{~m}$ & 16 & Meio-campo & $+0,25 \mathrm{D}$ & & $-0,25 \mathrm{D}$ & \\
\hline 27 & $\mathrm{~m}$ & 16 & Atacante & Plano & & $+0,25 \mathrm{D}$ & \\
\hline 28 & $\mathrm{~m}$ & 16 & M eio-campo & Plano & $-0,50 \mathrm{D}$ & Plano & $-0,50 \mathrm{D}$ \\
\hline 29 & $\mathrm{~m}$ & 16 & Goleiro & $+0,50 \mathrm{D}$ & & Plano & \\
\hline 30 & $\mathrm{~m}$ & 16 & Defesa & $-0,50 \mathrm{D}$ & $-0,50 \mathrm{D}$ & Plano & $-0,75 \mathrm{D}$ \\
\hline 31 & $\mathrm{~m}$ & 16 & Goleiro & Plano & $-0,50 \mathrm{D}$ & & \\
\hline 32 & $\mathrm{~m}$ & 16 & Atacante & Plano & & $-1,00 \mathrm{D}$ & \\
\hline 33 & $\mathrm{~m}$ & 16 & Atacante & $-0,25 \mathrm{D}$ & $-0,50 \mathrm{D}$ & $-0,50 \mathrm{D}$ & $-1,00 \mathrm{D}$ \\
\hline 34 & $\mathrm{~m}$ & 29 & Meio-campo & $+0,25 \mathrm{D}$ & $-0,50 \mathrm{D}$ & Plano & $-0,50 \mathrm{D}$ \\
\hline 35 & $\mathrm{~m}$ & 29 & Lateral & Plano & $-0,25 \mathrm{D}$ & $-0,25 \mathrm{D}$ & \\
\hline 36 & $\mathrm{~m}$ & 27 & M eio-campo & $-0,25 \mathrm{D}$ & & Plano & $-0,25 \mathrm{D}$ \\
\hline 37 & $\mathrm{~m}$ & 24 & Lateral & $-0,25 \mathrm{D}$ & & $-0,50 \mathrm{D}$ & \\
\hline 38 & $\mathrm{~m}$ & 20 & Defesa & & & Plano & $-0,25 \mathrm{D}$ \\
\hline 39 & $\mathrm{~m}$ & 23 & Goleiro & $-0,50 \mathrm{D}$ & $-0,25 \mathrm{D}$ & $-0,25 \mathrm{D}$ & $-0,25 \mathrm{D}$ \\
\hline 40 & $\mathrm{~m}$ & 29 & Meio-campo & Plano & $-0,50 \mathrm{D}$ & Plano & \\
\hline 41 & $\mathrm{~m}$ & 28 & Lateral & Plano & $-0,50 \mathrm{D}$ & Plano & $-0,50 \mathrm{D}$ \\
\hline 42 & $\mathrm{~m}$ & 25 & Lateral & & & $+0,25 \mathrm{D}$ & \\
\hline 43 & $\mathrm{~m}$ & 24 & Goleiro & Plano & $-0,50 \mathrm{D}$ & Plano & $-0,25 \mathrm{D}$ \\
\hline 44 & $\mathrm{~m}$ & 22 & Atacante & $-0,50 \mathrm{D}$ & $-0,50 \mathrm{D}$ & $-0,50 \mathrm{D}$ & $-0,25 \mathrm{D}$ \\
\hline 45 & $\mathrm{~m}$ & 21 & Atacante & $-0,25 \mathrm{D}$ & & & \\
\hline 46 & $\mathrm{~m}$ & 21 & Goleiro & Plano & $-0,50 \mathrm{D}$ & Plano & $-0,50 \mathrm{D}$ \\
\hline 47 & $\mathrm{~m}$ & 22 & Atacante & Plano & $-0,25 \mathrm{D}$ & $-0,25 \mathrm{D}$ & \\
\hline 48 & $\mathrm{~m}$ & 21 & Atacante & $-0,75 \mathrm{D}$ & & $-0,50 \mathrm{D}$ & $-0,25 \mathrm{D}$ \\
\hline 49 & $\mathrm{~m}$ & 23 & Defesa & Plano & & Plano & $-0,25 \mathrm{D}$ \\
\hline 50 & $\mathrm{~m}$ & 33 & Atacante & $-1,25 \mathrm{D}$ & & $-1,50 \mathrm{D}$ & $-0,25 \mathrm{D}$ \\
\hline 51 & $\mathrm{~m}$ & 23 & Defesa & $-0,25 \mathrm{D}$ & & $-0,50 \mathrm{D}$ & \\
\hline 52 & $\mathrm{~m}$ & 22 & Defesa & $-0,25 \mathrm{D}$ & Plano & $+1,00 \mathrm{D}$ & $-0,50 \mathrm{D}$ \\
\hline 53 & $\mathrm{~m}$ & 27 & Meio-campo & Plano & $-0,50 \mathrm{D}$ & Plano & $-0,50 \mathrm{D}$ \\
\hline 54 & $\mathrm{~m}$ & 23 & Defesa & $-0,25 \mathrm{D}$ & $-0,50 \mathrm{D}$ & $-0,25 \mathrm{D}$ & Plano \\
\hline 55 & $\mathrm{~m}$ & 24 & M eio-campo & $-1,00 \mathrm{D}$ & $-0,50 \mathrm{D}$ & $-0,75 \mathrm{D}$ & $-0,50 \mathrm{D}$ \\
\hline 56 & $\mathrm{~m}$ & 21 & Meio-campo & Plano & $-0,25 \mathrm{D}$ & Plano & $-0,25 \mathrm{D}$ \\
\hline
\end{tabular}


Em uma goleira, de 16 anos e cor negra, foi encontrado em OD $+0,75^{\circ}$ dioptria esférica e astigmatismo de $-2,50^{\circ}$ e em $\mathrm{OE}+0,50^{\circ} \mathrm{DE}$ e astigmatismo de $-1,75^{\circ} \mathrm{DC}$. Entretanto, os níveis de pressão intra-ocular foram considerados suspeitos devido à idade e à raça $(18,4$ e $17,8 \mathrm{mmHg}$ em $\mathrm{OD}$ e OE, respectivamente).

Em uma atacante, de origem japonesa, foi constatada catarata puntata congênita.

Portanto, ficou comprovado neste estudo que a maioria das alterações na acuidade visual dos atletas avaliados se deveu a vícios de refração (em ambos os olhos ou unilateral, levando às vezes à ambliopia).

Contudo, vale a pena ressaltar que devem ser investigados aqueles casos em atletas, que, apesar de se apresentarem sem vícios de refração, demonstraram acuidade visual abaixo do esperado e/ou pressão intra-ocular borderline.

O presente estudo comprovou o importante papel social da oftalmologia dentro de uma instituição desportiva, como medida preventiva na possível identificação de alterações oftalmológicas que possam comprometer o desempenho técnico e, sobretudo, para preservar a integridade ocular em seus atletas.

\section{AGRADECIMENTOS}

Gostaríamos de agradecer ao professor de língua inglesa Humberto Blancato por sua colaboração na redação do Abstract.

\section{REFERÊNCIAS}

1. Carvalho DM, Gonçalves P. Visão periférica e futebol (testes e treinamento). 1 ạ ed. Goiânia: Editora Grafopel, 1997.
2. Stock JG, Cornell FM. Prevention of sports related eye injury. Am Fam Physician 1991;44:515-20.

3. Fong LP. Sports-related eye injuries. Med J Aust 1994;160:743-7.

4. Marees H. Performance diagnosis of the locomotor system and sensory organs? Sportverletz Sportschaden 1989;3:53-7.

5. MacEwen CJ. Sports associated eye injury: a casualty department survey. Br J Ophthalmol 1987;71:701-5.

6. Barrell GV, Cooper PJ, Elkington AR, MacFadyen JM, Powell RG, Tormey P. Squash ball to eye ball: the likelihood of squash players in occurring an eye injury. Br Med J 1981;893-5.

7. Filipe JA, Barros H, Castro-Correia J. Sports related ocular injuries. A three year follow-up study. Ophthalmology 1997;104:313-8.

8. Mizusawa K, Sweeting RL, Knouse SB. Comparative studies of color fields, visual acuity fields and movement perception limits among varsity athletes and non-varsity groups. Percept Mot Skills 1983;56:88792.

9. Erie JC. Eye injuries: prevention, evaluation, and treatment. Phys Sports Med 1991;19:108-22.

10. Larrison WI, Hersh PS, Kunzweiler T, Shingleton BJ. Sport-related ocular trauma. Ophthalmology 1990;97:1265-9.

11. Strahlman E, Sommer A. The epidemiology of sports-related ocular trauma. Int Ophthalmol Clin 1988;28:199-202.

12. Vinger PF. The eye and sports medicine. In: Tasman W, editor. Duane's clinical ophthalmology. Chap 45:5, 1994

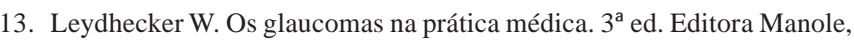
1980.

14. Vaughan D, Asbury T. Oftalmologia geral. $2^{\mathfrak{a}}$ ed. Ateneu, 1983.

15. Verdaguer J, Rojas B, Ibanez S, Leghuga M, Schmidt A. Desprendimiento retinal por pelotazo en el juego del fútbol. Arch Chil Oftalmol 1981;38:31-6.

16. A Joint Statement of the American Academy of Pediatrics and the American Academy of Ophthalmology, February 1995 and 1996. Protective eyewear for young athletes. Ophthalmology 1996;103:1325-8. 